\title{
Exome sequencing reveals HINT1 mutations as a cause of distal hereditary motor neuropathy
}

\author{
Hui Zhao ${ }^{1,2}$, Valérie Race ${ }^{3}$, Gert Matthijs ${ }^{3}$, Peter De Jonghe ${ }^{4,5,6}$, Wim Robberecht ${ }^{1,7,8}$, Diether Lambrechts ${ }^{1,2}$ \\ and Philip Van Damme $e^{\star, 1,7,8}$
}

Distal hereditary motor neuropathies (dHMNs) are a heterogenous group of genetic disorders with length-dependent degeneration of motor axons. Obtaining a genetic diagnosis in patients with $\mathrm{dHMN}$ remains challenging. We performed exome sequencing in a diagnostic setting in 12 patients with a clinical diagnosis of dHMN. Potential disease-causing variants in genes associated with dHMN and other forms of inherited neuropathies/motor neuron diseases were validated using Sequenom. The coverage in the genes studied was $>95 \%$ with an average coverage of $>50$ times. In none of the patients a mutations was found in genes previously reported to be associated with dHMN. However, in 2/12 patients a recessive mutation in histidine triad nucleotide binding protein 1 (HINT1, recently discovered as a cause of axonal neuropathy with neuromyotonia) was identified. Our results demonstrate the diagnostic value of exome sequencing for patients with inherited neuropathies. The phenotypic spectrum of recessive mutations in HINT1 includes dHMN. HINT1 should be added to the list of genes to check for in dHMN.

European Journal of Human Genetics (2014) 22, 847-850; doi:10.1038/ejhg.2013.231; published online 9 October 2013

Keywords: distal hereditary motor neuropathies; exome sequencing; HINT1

\section{INTRODUCTION}

Hereditary neuropathies are a clinically and genetically heterogenous group of disorders. ${ }^{1,2}$ Mutations in $>50$ genes have been identified to date and the inheritance pattern can be autosomal dominant, recessive or X-linked. ${ }^{3}$ The most common clinical phenotype is Charcot-Marie-Tooth (CMT) disease characterized by a lengthdependent sensorimotor neuropathy with progressive distal weakness, muscle atrophy, sensory loss and foot deformities. ${ }^{4-6}$ The distal hereditary motor neuropathies (dHMNs) are much rarer and present with a progressive distal motor weakness without sensory abnormalities. ${ }^{7,8}$ The disease usually starts in the lower limbs. Unusual presentations include dHMN with onset in the upper limbs or with vocal cord or diaphragm paralysis. Despite the degeneration of motor axons in the peripheral nerves, some patients have pyramidal signs due to upper motor neuron involvement. There is clinical and genetic overlap with the axonal form of CMT (CMT2) and with some motor neuron disorders (MNDs). ${ }^{7,9}$ The inheritance pattern of dHMN can also be autosomal dominant, autosomal recessive or X-linked. A significant proportion of dHMN patients will have a negative family history and can be classified as apparently sporadic cases. Mutations in the genes HSPB1, HSPB8, BSCL2, IGHMBP2, SETX, GARS, DYNC1H1, DCTN1, ATP7A, TRPV4, REEP1 and SLC5A7 explain $<20 \%$ of cases. Hence, $80 \%$ of dHMN is caused by mutations in as yet undiscovered genes. Obtaining a genetic diagnosis in dHMN patients in daily clinical practice remains a challenge.
We therefore performed exome sequencing in 12 patients with a clinical diagnosis of dHMN and looked for mutations in genes known to be associated with dHMN, but also checked CMT- and MNDcausing genes. ${ }^{7}$

\section{METHODS}

Patient selection

Patients with a slowly progressive pure motor length-dependent neuropathy followed at the neuromuscular reference center of the university hospital in Leuven with a clinical diagnosis of dHMN could participate in our study that aimed to better diagnose these patients after signing an informed consent. As a part of their genetic work-up, most patients had already undergone genetic testing for some of the genes involved in dHMN. For most of the patients, mutations in SMN1 had been excluded as well.

Exome sequencing and bioinformatics analysis

DNA was extracted from blood using standard techniques. The exomes were captured using the TruSeq exome enrichment kit (Illumina, San Diego, CA, USA). A $2 \times 100$ base pair paired-end sequencing was performed with the TruSeq SBS kit on an in-house HiSeq 2000 sequencer (Illumina). For all the exomes, BWA ${ }^{10}$ was used to align the raw reads to the human reference genome (NCBI Build 37/hg19) using default parameters. PCR duplicates were removed with Picard Mark Duplicates (http://picard.sourceforge.net). Base recalibration, local realignment, around small insertions/deletions (indels) and single-nucleotide variant calling were performed using the Genome Analysis ToolKit (GATK v1.0.4487). ${ }^{11}$ Substitutions were called using the Genome Analysis ToolKit (GATK v.1.0.4487) Unified Genotyper, ${ }^{11}$ while small

${ }^{1}$ Vesalius Research Center, VIB, Leuven, Belgium; ${ }^{2}$ Laboratory for Translational Genetics, Department of Oncology, KU Leuven, Leuven, Belgium; ${ }^{3}$ Laboratory for Molecular Diagnostics, Center for Human Genetics, KU Leuven, Leuven, Belgium; ${ }^{4}$ Neurogenetics Laboratory, Institute Born-Bunge, University of Antwerp, Antwerp, Belgium; ${ }^{5}$ Neurogenetics Group, VIB Department of Molecular Genetics, University of Antwerp, Antwerp, Belgium; ${ }^{6}$ Department of Neurology, Antwerp University Hospital, Antwerp, Belgium; ${ }^{7}$ Department of Experimental Neurology, Leuven Research Institute for Neurodegenerative Diseases (LIND), KU Leuven, Leuven, Belgium; ${ }^{8}$ Department of Neurology, University Hospital Leuven, Leuven, Belgium

${ }^{*}$ Correspondence: Professor P Van Damme, Neurology Department, University Hospital Leuven, Herestraat 49, Leuven 3000, Belgium. Tel: +32 16 344280 ; Fax: +32 344285; E-mail: philip.vandamme@uzleuven.be

Received 6 May 2013; revised 2 September 2013; accepted 4 September 2013; published online 9 October 2013 
insertions/deletions (indels) were detected using Dindel v1.01. ${ }^{12}$ Substations and indels in the coding region of the captured regions were selected for further analysis.

Commonly occurring substitutions and indels are unlikely to be disease causing and therefore eliminated using a set of publicly available variant data sets. These databases included: all common SNPs from dbSNP version 132 (defined as SNPs with a minor allele frequency of $>1 \%$ ), variants identified in the March 2012 release of the 1000 Genomes Project, common SNPs from 126 HapMap3 individuals in 11 populations provided by Affymetrix (Santa Clara, CA, USA) and variants identified in 46 HapMap individuals using the Complete Genomics whole-genome sequencing technology. Stepwise filtering was applied on all variants of each exome. As such, we were able to detect all novel variants in each patient. A list of these filtered variants, as well as the rough data, have been submitted to EGA (accession number EGAS00001000576). For diagnostic purposes, we looked for known disease-causing mutations in these lists of variants for each patient.

\section{Validation of genetic variants identified}

Standard Sequenom MassARRAY genotyping experiments were performed to validate candidate variants according to the manufacturer's conditions, a strategy previously used. ${ }^{13}$ Variants that failed to be successfully genotyped in the first round of validation were subsequently redesigned for a second attempt using a new set of Sequenom primers (e.g, by designing new extension primers that annealed on the other DNA strand as the extension primer from the first validation round).

\section{RESULTS}

We performed exome sequencing in 12 patients followed at the neuromuscular reference center of the university hospitals in Leuven with a clinical diagnosis of dHMN. All patients had undergone genetic testing to some extent as a part of their work-up, but the results were negative in all patients. None of the patients belonged to extended pedigrees. The 12 patients had a pure motor phenotype, reported no sensory complaints and had a normal sensory neurological examination. Some of them had pyramidal signs with hyperreflexia, but always without Babinski signs. None of the patients had vocal cord involvement. The clinical and electrodiagnostic findings of the patients are summarized in Table 1. Conduction blocks or other signs of demyelinization were absent in all patients. The sural sensory nerve action potential was normal in all patients.

The diagnostic value of exome sequencing in this cohort of genetically unexplained dHMN patients was evaluated. We looked for mutations in genes known to be associated with dHMN.
Since there is clinical overlap with other inherited neuropathies such as CMT and with some forms of MND, we also assessed whether mutations in genes underlying CMT or MND were present. The list of 97 genes studied can be found in Supplementary Table 1. This list includes all genes known to be associated with dHMN, CMT and other inherited neuropathies, but also other phenotypes with lower motor neuron involvement.

On average, all mappable reads covered $93.0 \%$ of the TruSeq captured regions, with an average coverage of $62.2 \times$. The total substitutions and indels count ranged from 51018 to 52805 and from 21807 to 34843 , respectively, in the different patients. Among these variants, on average, for each patient, 22587 subsititions and 498 indels are located in the coding region while 557 substitutions and 35 indels were detected as possible disease-causing mutations after eliminating commonly occurring variants. Within the 97 studied disease-causing genes, a coverage of $95.8 \%$ of the coding regions was obtained with an average coverage of $50.2 \times$, suggesting that exome sequencing can pick up coding mutations with a considerable sensitivity in the diagnostic setting (Supplementary Table 1 gives an overview of the list of all genes studied, with the coverage per gene).

First, we looked for known disease-causing mutations in genes known to be associated with dHMN. In none of the typical dHMNcausing genes known mutations were found, illustrating the low frequency of occurrence of mutations in each of these genes. Next, we also checked genes associated with other forms of inherited neuropathies or MND (Supplementary Table 1). Likewise, no pathogenic mutations were identified in genes underlying CMT, hereditary sensory and autonomic neuropathies (HSANs) or MND. In addition, we also looked for any variants that were not previously reported as causative mutations in the selected genes, as these variants could potentially be pathogenic. For genes with recessive inheritance only homozygous variants or compound heterozygous variants were taken into account and validated using Sequenom MassARRAY. In total, seven variants in five genes occurring in five patients could be validated. These variants are listed in Table 2, however, the pathogenicity of these variants remains uncertain.

Strikingly, in $2 / 12$ patients ( $\sim 17 \%$, a proportion close to the total proportion of explained dHMN cases in other cohorts) a recessive mutation in histidine triad nucleotide binding protein 1 (HINT1) was identified. In patient dHMN4 a homozygous c.250T $>\mathrm{C}$ (p.(Cys84Arg)) mutation was found, patient dHMN11 was

Table 1 Patient characteristics

\begin{tabular}{|c|c|c|c|c|c|c|c|c|c|}
\hline Patient ID & Sex & $\begin{array}{c}\text { Onset } \\
\text { age }\end{array}$ & $\begin{array}{l}\text { Onset } \\
\text { site }\end{array}$ & $\begin{array}{c}\text { UL } \\
\text { involvement }\end{array}$ & Reflexes & $\begin{array}{l}\text { Pes } \\
\text { cavus }\end{array}$ & $\begin{array}{c}\text { Inheritance } \\
\text { pattern }\end{array}$ & $\begin{array}{c}\text { Sural SNAP } \\
\text { amplitude }(\mu V)\end{array}$ & $\begin{array}{c}E D B C M A P \\
\text { amplitude ( } m V)\end{array}$ \\
\hline dHMN1 & $\mathrm{F}$ & 29 & UL and LL & Yes & Areflexia & Yes & $A D$ & 10 & 1.1 \\
\hline dHMN2 & $M$ & 45 & UL & Yes & Hyperreflexia & Yes & $A D$ & 15 & 3.7 \\
\hline dHMN3 & $\mathrm{F}$ & 26 & LL & Yes & Hyporeflexia & Yes & AR & 7.5 & 1.6 \\
\hline dHMN4 & $\mathrm{F}$ & 17 & LL & No & Hyporeflexia & Yes & Apparently sporadic & 20 & 2.8 \\
\hline dHMN5 & M & 1 & LL & Yes & Hyporeflexia & $+1-$ & AR & 19 & 0.5 \\
\hline dHMN7 & M & 17 & LL & No & Hyperreflexia & Yes & $A D$ & 16 & 5.3 \\
\hline dHMN8 & $\mathrm{F}$ & 54 & $\mathrm{LL}$ & No & Hyporeflexia & Yes & Apparently sporadic & 10 & 3.7 \\
\hline dHMN9 & M & 30 & LL & Yes & Hyporeflexia & Yes & $A D$ & 4 & 0.9 \\
\hline dHMN10 & M & 19 & $\mathrm{LL}$ & Yes & Hyperreflexia & Yes & Apparently sporadic & $>10$ & $<5$ \\
\hline dHMN11 & M & 28 & LL & No & Hyperreflexia & Yes & AR & 38 & NR \\
\hline dHMN12 & $\mathrm{F}$ & 16 & LL & Yes & Hyperreflexia & Yes & Apparently sporadic & 14 & NR \\
\hline dHMN13 & $\mathrm{F}$ & 1 & LL & Yes & Hyporeflexia & $+1-$ & Apparently sporadic & 13.1 & 0.1 \\
\hline
\end{tabular}

Abbreviations: F, female; M, male; LL, lower limbs; UL, upper limbs; AD, autosomal dominant; AR, autosomal recessive; SNAP, sensory nerve action potential; EDB CMAP, extensor digitorum brevis compound muscle action potential; NR, no response. 
compound heterozygous for the same p.C84R mutation and the c.341A $>$ G (p.(His114Arg)) mutation. These mutations were confirmed using standard Sequenom MassARRAY genotyping experiments. The non-consanguineous parents of these patients were asymptomatic and heterozygous carriers of the p.(Cys84Arg) or p.(His114Arg) mutation (Figure 1a). Both the p.(Cys84Arg) mutation and the p.(His114Arg) mutation were predicted to be damaging by SIFT (Craig Venter Institute, San Diego, CA, USA) and Polyphen-2 software (Harvard University, Cambridge, MA, USA) (with a score of 0.992 and 1.000 for p.(Cys84Arg) and p.(His114Arg), respectively). Both amino-acid substitutions were affecting a highly conserved residue (Figure 1b). Our data demonstrate that exome sequencing is useful for diagnostic purposes in patients with $\mathrm{dHMN}$ and reveal that recessive mutations in HINT1 are a cause of dHMN.

\section{DISCUSSION}

In this study, using exome sequencing for diagnostic purposes in patients with dHMN, we identified mutations in HINT1 in a considerable proportion of patients. Recessive loss-of-function mutations were recently reported ${ }^{14}$ in patients with a rare phenotype of axonal neuropathy with neuromyotonia ${ }^{15}$ and CMT. The gene was identified in a family with CMT with neuromyotonia and in a consanguineous family with CMT, but mutations in HINT1 were also observed in rare patients with autosomal recessive or sporadic CMT. Most patients from the original report had a sensorimotor axonal neuropathy that was associated with action myotonia in the hands and/or neuromyotonic discharges on needle electromyography. The p.(Cys84Arg) mutation was one of the loss-of-function mutations described in the original study. ${ }^{14}$ The patients described here had a phenotype of dHMN without evidence of sensory involvement or

Table 2 Novel variants in genes associated with inherited neuropathies

\begin{tabular}{|c|c|c|c|c|}
\hline Gene & Chromosome & Position & Mutation & Patient \\
\hline ATL1 & chr14 & 51098982 & c. $1587 C>A(p .(H i s 529 G \mid n))$ & dHMN9 \\
\hline DNMT1 & chr19 & 10250470 & c.3830G >A (p.(Arg1277GIn)) & dHMN4 \\
\hline KARS & chr16 & 75669656 & c.801T> G (p.(Phe267Leu)) & dHMN10 \\
\hline$P R X$ & chr19 & 40900171 & c. $4088 \mathrm{G}>\mathrm{A}$ (p.(Ser1363Asn)) & dHMN9 \\
\hline$P R X$ & chr19 & 40902776 & c. $1483 G>C(p .(G l u 495 G \mid n))$ & $\begin{array}{l}\text { dHMN9, } \\
\text { dHMN12 }\end{array}$ \\
\hline SPG11 & chr15 & 44890530 & c.3934A > C (p.(Thr1312Pro)) & dHMN3 \\
\hline SPG11 & chr15 & 44888316 & c.4399G >C (p.(Asp1467Pro)) & dHMN3 \\
\hline
\end{tabular}

Abbreviations: ATL1, Atlastin GTPase 1; DNMT1, DNA (Cytosine-5-)-Methyltransferase 1; KARS, Lysyl-tRNA Synthetase; PRX, Periaxin; SPG11, Spastic Paraplegia 11. neuromyotonia and extend the phenotypic spectrum of HINT1 mutations. These findings warrant the inclusion of HINT1 to the list of genes to be tested in the diagnostic work-up of patients with dHMN. HINT1 binds and hydrolyses adenosine 5'-monophosphoramidate substrates. It is a ubiquitously expressed haploinsufficient tumor suppressor gene ${ }^{16}$ that promotes apoptosis, ${ }^{17}$ inhibits the transcriptional activity of beta-catenin/TCF4, USF2 and NFkappaB, and inhibits the expression of endogenous cyclin D1 and TGFbeta2. ${ }^{18}$ How loss-of-function mutations in HINT1 can cause an axonal neuropathy is unknown. HINT1 knockout mice have been shown to have an increased susceptibility to spontaneous tumor development and to tumor induction. Whether these mice have an axonal neuropathy remains unexplored. ${ }^{16}$

Over the last few years, many studies have shown the value of exome sequencing for gene discovery purposes in neurological disorders. ${ }^{19-23}$ Next-generation sequencing technologies have also accelerated gene discovery in patients with $\mathrm{dHMN},{ }^{9,24} \mathrm{CMT}^{25,26}$ or other inherited neuropathies. ${ }^{27-29}$ More recently, the use of exome sequencing for diagnostic purposes in genetic disorders with a high degree of genetic heterogeneity such as CMT is being explored. ${ }^{30,31}$ Although the prize of exome sequencing is declining, the bioinformatic tools for analysis have improved and the coverage of the sequencing appears to be sufficiently high for use in the diagnostic setting, several technical and ethical challenges remain. ${ }^{32}$ In particular, establishing the pathogenicity of newly identified genetic variants is often difficult and the possibility of finding unrelated finding requires further attention. A focused analysis of a predefined set of genes, as was done in this study, may circumvent the latter issue. In our study in dHMN patients, we also identified novel variants in genes known to be associated with various forms of inherited neuropathies or related neuromuscular disorders. However, the pathogenic nature of these variants remains uncertain, as the patients did not belong to extended pedigrees allowing segregation analysis.

Several studies have shown that exome sequencing is a reliable firsttier method to screen for mutations in coding regions of a large set of genes in patients with genetically heterogenous neuromuscular disorders. $^{31,33-35}$ In our study, we showed that the coverage of a predefined list of genes associated with $\mathrm{dHMN}$ and overlapping disorders was sufficiently high to use exome sequencing as an initial diagnostic approach. In addition, the combined use of Sequenom MassARRAY to validate the genetic variants identified appeared to be a fast and powerful strategy. A screening for mutations in genes associated with dHMN did not reveal any hits, confirming that each of the genes is only responsible for a small proportion of dHMN. a

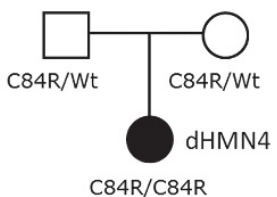

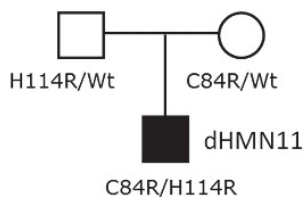

b

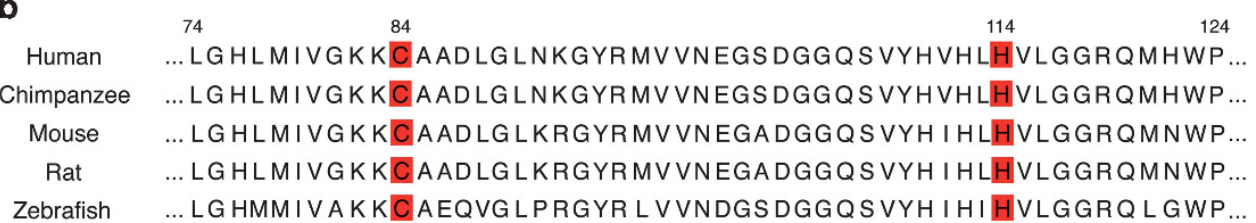

Figure 1 HINT1 mutations observed in dHMN patients. (a) Homozygous p.(Cys84Arg) and compound heterozygous p.(Cys84Arg) and p.(His114Arg) mutations observed in patients dHMN4 and dHMN11. (b) Conservation of residues 84 and 114 in HINT1 protein across species (marked in red). 
The analysis in our study was not limited to genes stringently linked to dHMN. The study of a wider set of genes associated with other forms of inherited neuropathies or more complex phenotypes including neuropathy allowed us to identify recessive mutations in HINT1 as a cause of dHMN. This comprehensive approach appears to be an advantage of the use of exome sequencing in clinical practice.

\section{CONFLICT OF INTEREST}

The authors declare no conflict of interest.

\section{ACKNOWLEDGEMENTS}

This work was supported by the VIB Tech Watch Fund, by a grant from the KU Leuven (GOA/11/014) and by the Interuniversity Attraction Poles (IUAP) program P7/16 of the Belgian Federal Science Policy Office. PVD holds a clinical investigatorship of the FWO-Vlaanderen. HZ holds a postdoctoral fellowship of the FWO-Vlaanderen. WR is supported through the E von Behring Chair for Neuromuscular and Neurodegenerative Disorders.

1 Patzko A, Shy ME: Charcot-Marie-Tooth disease and related genetic neuropathies. Continuum 2012; 18: 39-59.

2 Boerkoel CF, Takashima H, Garcia CA et al: Charcot-Marie-Tooth disease and related neuropathies: mutation distribution and genotype-phenotype correlation. Ann Neurol 2002; 51: 190-201.

3 Kaplan JC: The 2012 version of the gene table of monogenic neuromuscular disorders. Neuromuscul Disord 2011; 21: 833-861.

4 Barisic N, Claeys KG, Sirotkovic-Skerlev M et al: Charcot-Marie-Tooth disease: a clinico-genetic confrontation. Ann Hum Genet 2008; 72: 416-441.

5 Zuchner S, Vance JM: Molecular genetics of autosomal-dominant axonal CharcotMarie-Tooth disease. Neuromol Med 2006; 8: 63-74.

6 Pareyson D, Marchesi C: Diagnosis, natural history, and management of CharcotMarie-Tooth disease. Lancet Neurol 2009; 8: 654-667.

7 Rossor AM, Kalmar B, Greensmith L, Reilly MM: The distal hereditary moto neuropathies. J Neurol Neurosurg Psychiatry 2012; 83: 6-14.

8 Irobi J, De Jonghe P, Timmerman V: Molecular genetics of distal hereditary motor neuropathies. Hum Mol Genet 2004; 13 Spec No 2: R195-R202.

9 Beetz C, Pieber TR, Hertel N et al: Exome sequencing identifies a REEP1 mutation involved in distal hereditary motor neuropathy type V. Am J Hum Genet 2012; 91 : 139-145.

$10 \mathrm{Li} \mathrm{H}$, Durbin R: Fast and accurate long-read alignment with Burrows-Wheele transform. Bioinformatics 2010; 26: 589-595.

11 McKenna A, Hanna M, Banks E et al: The Genome Analysis Toolkit: a MapReduce framework for analyzing next-generation DNA sequencing data. Genome Res 2010; 20: 1297-1303.

12 Albers CA, Lunter G, MacArthur DG, McVean G, Ouwehand WH, Durbin R Dindel: accurate indel calls from short-read data. Genome Res 2011; 21: 961-973.

13 Herdewyn $\mathrm{S}$, Zhao $\mathrm{H}$, Moisse $\mathrm{M}$ et al: Whole-genome sequencing reveals a coding non-pathogenic variant tagging a non-coding pathogenic hexanucleotide repeat expansion in C9orf72 as cause of amyotrophic lateral sclerosis. Hum Mol Genet 2012; 21: 2412-2419

14 Zimon M, Baets J, Almeida-Souza L et al: Loss-of-function mutations in HINT1 cause axonal neuropathy with neuromyotonia. Nat Genet 2012; 44: 1080-1083.

15 Hahn AF, Parkes AW, Bolton CF, Stewart SA: Neuromyotonia in hereditary moto neuropathy. J Neurol Neurosurg Psychiatry 1991; 54: 230-235.

16 Li H, Zhang Y, Su T, Santella RM, Weinstein IB: Hint1 is a haplo-insufficient tumor suppressor in mice. Oncogene 2006; 25: 713-721.

17 Weiske J, Huber O: The histidine triad protein Hint1 triggers apoptosis independent of its enzymatic activity. J Biol Chem 2006; 281: 27356-27366.

18 Wang L, Li H, Zhang Y, Santella RM, Weinstein IB: HINT1 inhibits beta-catenin/TCF4 USF2 and NFkappaB activity in human hepatoma cells. Int J Cancer 2009; 124 1526-1534.

19 Zimprich A, Benet-Pages A, Struhal W et al: A mutation in VPS35, encoding a subunit of the retromer complex, causes late-onset Parkinson disease. Am J Hum Genet 2011 89: 168-175.

20 Vilarino-Guell C, Wider C, Ross OA et al: VPS35 mutations in Parkinson disease. Am J Hum Genet 2011; 89: 162-167.

21 Noskova L, Stranecky V, Hartmannova $\mathrm{H}$ et al: Mutations in DNAJC5, encoding cysteine-string protein alpha, cause autosomal-dominant adult-onset neuronal ceroid lipofuscinosis. Am J Hum Genet 2011; 89: 241-252.

22 Johnson JO, Mandrioli J, Benatar M et al: Exome sequencing reveals VCP mutations as a cause of familial ALS. Neuron 2010; 68: 857-864.

23 Wang JL, Yang X, Xia $\mathrm{K}$ et al: TGM6 identified as a novel causative gene of spinocerebellar ataxias using exome sequencing. Brain 2010; 133 3510-3518.

24 Barwick KE, Wright J, Al-Turki S et al: Defective presynaptic choline transport underlies hereditary motor neuropathy. Am J Hum Genet 2012; 91: 1103-1107.

25 Kennerson ML, Yiu EM, Chuang DT et al: A new locus for X-linked dominant CharcotMarie-Tooth disease (CMTX6) is caused by mutations in the pyruvate dehydrogenase kinase isoenzyme 3 (PDK3) gene. Hum Mol Genet 2013; 22: 1404-1416.

26 Rinaldi C, Grunseich C, Sevrioukova IF et al: Cowchock syndrome is associated with mutation in apoptosis-inducing factor. Am J Hum Genet 2012; 91: 1095-1102.

27 Klein CJ, Botuyan MV, Wu Y et al: Mutations in DNMT1 cause hereditary sensory neuropathy with dementia and hearing loss. Nat Genet 2011; 43: 595-600.

28 Ishiura H, Sako W, Yoshida M et al: The TRK-fused gene is mutated in hereditary motor and sensory neuropathy with proximal dominant involvement. Am J Hum Genet 2012 91: 320-329.

29 Edvardson S, Cinnamon Y, Jalas C et al: Hereditary sensory autonomic neuropathy caused by a mutation in dystonin. Ann Neurol 2012; 71: 569-572.

30 Ku CS, Cooper DN, Polychronakos C, Naidoo N, Wu M, Soong R: Exome sequencing: dual role as a discovery and diagnostic tool. Ann Neurol 2012; 71: 5-14.

31 Montenegro G, Powell E, Huang J et al: Exome sequencing allows for rapid gene identification in a Charcot-Marie-Tooth family. Ann Neurol 2011; 69: 464-470.

32 Nelen M, Veltman JA: Genome and exome sequencing in the clinic: unbiased genomic approaches with a high diagnostic yield. Pharmacogenomics 2012; 13: 511-514.

33 Dias C, Sincan M, Cherukuri PF et al: An analysis of exome sequencing for diagnostic testing of the genes associated with muscle disease and spastic paraplegia. Hum Mutat 2012; 33: 614-626.

34 Hanchard N, Murdock D, Magoulas P et al: Exploring the utility of whole-exome sequencing as a diagnostic tool in a child with atypical episodic muscle weakness. Clin Genet 2013; 83: 457-461.

35 Sailer A, Scholz SW, Gibbs JR et al: Exome sequencing in an SCA14 family demonstrates its utility in diagnosing heterogeneous diseases. Neurology 2012; 79: 127-131.

Supplementary Information accompanies this paper on European Journal of Human Genetics website (http://www.nature.com/ejhg) 